\title{
ESTUDO DO GANHO DE PESO E DURAÇÃO DA INTERNAÇÃO DO RECÉM-NASCIDO PRÉ-TERMO DE BAIXO PESO COM A UTILIZAÇÃO DO MÉTODO CANGURU
}

\author{
TRIAL GAIN OF WEIGHT AND HOSPITAL LENGTH STAY OF THE LOW BIRTH WEIGHT PRETERM INFANT IN \\ ASSISTANCE FOR KANGAROO MOTHER CARE
}

\section{ESTUDIO DEL AUMENTO DE PESO Y DURACIÓN DE LA INTERNACIÓN DEL RECIÉN NACIDO PRÉ-TERMO COM BAJO PESO CON LA UTILIZACIÓN DEL MÉTODO CANGURO}

\section{Márcia Aparecida Giacomini Rodrigues ${ }^{1}$, Maria Aparecida Tedeschi Cano ${ }^{2}$}

RESUMO: O objetivo deste estudo foi avaliar se o Método Canguru (MC) interfere no ganho de peso e na duração do tempo de internação do recém-nascido pré-termo de baixo peso (RNPTBP). Para isto foi realizado um estudo analítico retrospectivo, através da avaliação de prontuários médicos de 60 RNPTBP com peso de nascimento menor que $2000 \mathrm{~g}$ que receberam assistência pelo $M C$, por um período de 2 horas por dia e, 60 RNPTBP que receberam assistência pelo Método Tradicional (MT), admitidos na unidade de tratamento intensivo neonatal e unidade de prematuros de uma maternidade privada na cidade de Ribeirão Preto-SP, comparando-se o ganho de peso e a duração do período de internação. Não encontramos diferenças estatisticamente significativas em relação ao ganho de peso dos RNPTBP assistidos pelo $M C$ em relação aos RNPTBP assistidos pelo MT, 15,8 e 14,9g/dia, respectivamente. Em relação ao tempo de internação, também não encontramos diferenças estatisticamente significativas, 27,3 e 26,2 dias para os RNPTBP no MC e MT, respectivamente, porém na estratificação da amostra, os RNPTBP com idade gestacional $<30$ semanas ou peso de nascimento $<1500 \mathrm{~g}$ receberam alta 3 dias mais cedo no MC.Concluímos que o MC, nas condições deste estudo, parece não interferir significativamente no ganho de peso e no tempo de internação do RNPTBP. Ressaltamos que a utilização do $M C$ na assistência ao RNPTBP é um modelo viável, mesmo para instituição de saúde privada.

PALAVRAS CHAVE: Método canguru; Prematuro; Ganho de peso; Tempo de internação.

ABSTRACT: The aim of this study was to evaluate if the Kangaroo Mother Care (KMC) interferes in the gain of weight and in the hospital length stay of the low birth weight preterm newborn (LWBPTN). For this, it was realized an analytic retrospective study, through the evaluation of the medical records of 60 LWBPTN with born weight less than $2000 \mathrm{~g}$ that received assistance by KMC, for a period of 2 hours per day and, 60 LWBPTN that received assistance by the Traditional Method of Care (TMC), admitted in the unity of neonatal intensive care and unity of premature of a private maternity in the city of Ribeirão Preto-SP, Brazil, comparing the gain of weight and the hospital length stay. We did not find differences statistically significant in relation to the gain of weight of the LWBPTN assisted by KMC, in relation to LWBPTN assisted by the TMC, 15,8 and 14,9g/per day, respectively. In relation to the time of admission, we also did not find differences statistically significant, 27,3 and 26,2 days, for the LWBPTN in the KMC and $\mathrm{TMC}$, respectively, although in the stratification of the sample, the LBWPTN with pregnancy age $<30$ weeks or birth weight $<1500 \mathrm{~g}$ left the hospital 3 days earlier in the $\mathrm{KMC}$. It was concluded that the $\mathrm{KMC}$, in the conditions of this study, it seems not to interfere significatively in the gain of weight and in the time of admission of LWBPTN. We emphasize that the utilization of KMC in the assistance to the LWBPTN of low weight is a viable model, even for the private health institution.

KEY WORDS: Kangaroo mother care; Premature; Weight Gain; Length of stay.

RESUMEN: El objetivo de este estudio fue evaluar si el Método Canguro (MC) interfiere en el aumento de peso y en la duración del tiempo de internación del recién nacido pre-termo de bajo peso (RNPTBP). Para esto, fue realizado un estudio analítico retrospectivo, a través de la evaluación de informes médicos de 60 RNPTBP con menos de $2000 \mathrm{~g}$ de peso al nacer, que recibieron asistencia del MC, en un periodo de 2 horas diarias y de, 60 RNPTBP que recibieron asistencia a través del método tradicional (MT), admitidos en la unidad de tratamiento intensivo neonatal y unidad de prematuros de una maternidad privada en la ciudad de Ribeirão Preto-SP, Brasil, comparando el aumento de peso y la duración del tiempo de internación entre ellos. No se encontró distinción estadística significativa con relación al aumento de peso de los RNPTBP asistidos por el MC y a los RNPTBP asistidos por ele MT que fue de 15,8 y 14,9 gramos al día, respectivamente. Con relación al tiempo de internación, tampoco se encontró distinción estadística significativa que fue de 27,3 y 26,2 días promedios para los RNPTBP en el MC y MT respectivamente. Pero, en la estratificación de demostración, los RNPTBP con edad de gestación menor que treinta semanas o peso de nacimiento menor que ciento cincuenta gramos, estos recibieron

\footnotetext{
Enfermeira/Mestre em Promoção à Saúde pela Universidade de Franca. Franca/SP

2 Livre Docente/Orientadora do Curso de Pós-Graduação de Promoção à Saúde da Universidade de Franca. Franca/SP.E-mail: decano@netsite.com.br
} 
baja del hospital tres días más temprano cuando utilizado el MC. Se puede concluir que el MC, en las condiciones de este estudio, parece no intervenir significativamente en el aumento de peso y en el tiempo de internación del RNPTBP pero se debe resaltar que la utilización del MC en la asistencia al

\section{INTRODUÇÃO}

A prematuridade tem se tornado cada vez mais um problema de saúde pública. Além das patologias específicas da gravidez que desencadeiam o trabalho de parto prematuro, o desgaste físico e emocional da vida moderna tem interferido no ciclo gravídico, desencadeando o parto prematuro. Paralelamente, os avanços tecnológicos na área médica, têm propiciado a sobrevida de bebês cada vez menores e mais imaturos. (CANO et al, 2001; DOLE et al, 2003)

A prematuridade predispõe a complicações que interferem na qualidade de vida da criança e do núcleo familiar. $O$ desafio da equipe de saúde que assiste a esse recém-nascido prematuro de baixo peso é possibilitar sua inserção na família $e$ sociedade, em condições cada vez melhores, diminuindo ao máximo as seqüelas advindas da sua condição de prematuro, como também, pelo longo tempo de tratamento intensivo ao qual tenha sido submetido, que o predispõe a complicações audiovisuais neurológicas, pulmonares e ao risco de infecções hospitalares. (CANO et al, 2001; WAGNER et al, 1990; CUST et 2003).

Medidas que melhorem a qualidade da assistência a esses recém-nascidos prematuros devem ser implementadas visando diminuir complicações futuras. Neste aspecto, o Método Canguru apresenta inúmeros benefícios para o recém-nascido pré-termo de baixo peso e sua família, constituindo-se em recomendação de organizações científicas e governamentais para a assistência integral e humanizada em unidade neonatal (WHO, 2004).

A literatura aponta que o Método Canguru promove menor incidência de hipotermia; que a alta hospitalar com aleitamento materno exclusivo é mais comum nos bebês tratados com o método canguru; que as mães expressam preferência pelo Método Canguru e o custo hospitalar diminui com este método. Quanto ao ganho diário de peso que significa importante variável para a evolução do bebê prematuro e, sua conseqüente alta hospitalar, defende que o método canguru implica num ganho de peso diário maior e num tempo para a alta hospitalar menor que no tratamento convencional (CATTANEO et al, 1998). Alguns estudos, também apontam para uma redução no tempo de hospitalização dos bebês submetidos ao Método Canguru (CHARPAK et al, 2004; LEGAULT \& GOULET, 1995).
RNPTBP es método viable aunque sea en una institución privada de salud.

PALABRAS CLAVE: Método Canguro; Prematuro; Aumento de peso; Tiempo de internación.

Por outro lado, outros autores citam que não encontraram diferenças no ritmo diário de ganho de peso e adesão ao aleitamento materno (SLOAN et al, 1994), fatos possivelmente explicáveis pela forte política de incentivo ao aleitamento materno existente em seu local de estudo.

No entanto, uma das principais dificuldades para a implantação do Método Canguru é a adesão da equipe de saúde que atua com esses recémnascidos prematuros. Para tanto, uma série de medidas estão sendo implementadas pelo Ministério da Saúde, que desde 1999 implantou em todo Brasil a "Norma de atenção humanizada ao recém-nascido prematuro de baixo peso - Método Canguru" e, desde então, não tem medido esforços na tentativa de difundir o Método Canguru e capacitar os profissionais envolvidos na assistência ao recém nascido prematuro de baixo peso. Por outro lado, a adesão dos profissionais pode ser facilitada se as evidências das vantagens do Método Canguru forem consolidadas (MINISTĖRIO DA SAÚDE, 2002).

Porém, não basta aos profissionais de saúde conhecer, estarem disponíveis e aceitarem a realização do Método Canguru, deve haver uma mudança de comportamento da equipe e da filosofia da instituição no sentido de buscar a humanização da assistência ao recém-nascido e sua família (GENOVESE, 2001).

Sabe-se que o ganho de peso do recém nascido prematuro de baixo peso implica em melhoria de sua qualidade de vida e, conseqüentemente, aumenta a perspectiva da alta hospitalar, reduzindo os efeitos adversos da internação, inserindo-o mais rapidamente ao seu núcleo familiar (CATTANEO et al, 1998,).

O presente estudo teve por objetivo avaliar se o Método Canguru interfere no ganho de peso e na duração do tempo de internação do recém nascido prematuro de baixo peso.

\section{METODOLOGIA}

Estudo analítico retrospectivo, realizado por meio de avaliação de prontuários médicos de recémnascidos prematuros (idade gestacional < 37 semanas ao nascimento) e com peso de nascimento menor que $2000 \mathrm{~g}$, assistidos na unidade de terapia intensiva neonatal e berçário de uma maternidade privada da cidade de Ribeirão Preto.

A amostra foi dividida em dois grupos:

- Recém-nascidos prematuros que receberam assistência com o Método Canguru (RNPT/MC). 
Dados obtidos referente ao período de janeiro de 2002 a junho de 2004;

- Recém-nascidos prematuros que receberam assistência com o Método Tradicional (RNPT/MT). Dados obtidos referente ao mesmo período acima, para recém-nascidos pré-termo (RNPT) cujas mães optaram por não realizar o contato pele-a-pele e, em período retroativo que antecedeu a implantação do Método na instituição, até obter um número semelhante de sujeitos desejáveis para comparação estatística.

Os critérios de inclusão na amostra foram a prematuridade (idade gestacional ao nascimento $<37$ semanas), peso de nascimento $<2000 \mathrm{~g}$, estabilidade clínica, ausência de mal-formações congênitas, nenhuma intervenção cirúrgica e nenhum registro de ocorrência de sepse.

Os parâmetros avaliados foram o ganho de peso e tempo de internação. Outras variáveis também foram registradas para caracterização da amostragem, como idade materna, paridade, idade gestacional, peso ao nascimento, comprimento ao nascimento, sexo, valor do apgar de primeiro e quinto minutos, peso na alta e, diagnóstico clínico.

Para análise estatística foi utilizado o teste $t$ de student, um tipo de análise de variância (ANOVA) para comparar duas amostras independentes.

Este Estudo foi aprovado pelo Comitê de Ética em Pesquisa da Universidade de Franca, sob o protocolo 034/04 e com o consentimento da instituição.

\section{RESULTADOS}

Foram avaliados 120 prontuários de RNPT, dos quais $60(50 \%)$ receberam assistência pelo Método Canguru (MC) e 60 (50\%), receberam assistência pelo Método Tradicional (MT). Na amostra avaliada, em relação ao sexo, 62 (52\%) eram meninos e, 58 $(48 \%)$ eram meninas. Os valores para caracterização da amostra são apresentados em valores absolutos e porcentagem na Tabela 1 e como média \pm 0 desviopadrão na Tabela 2.

Tabela 1- Distribuição numérica e percentual, segundo o sexo, dos recém-nascidos pré-termo assistidos pelo Método Canguru e Método Tradicional, numa maternidade privada de Ribeirão Preto, no período de janeiro de 2002 a junho de 2004.

\begin{tabular}{lrr:r}
\hline Sexo & MC & MT & Total \\
\hline Masculino & $33(55 \%)$ & $29(48 \%)$ & $62(52 \%)$ \\
Feminino & $27(45 \%)$ & $31(52 \%)$ & $58(48 \%)$ \\
\hline
\end{tabular}

$\mathrm{MC}=$ método canguru; $\mathrm{MT}=$ método tradicional.

Tabela 2 - Caracterização da amostra dos recém-nascidos pré-termo assistidos pelo Método Canguru e Método Tradicional, numa maternidade privada de Ribeirão Preto, no período de janeiro de 2002 a junho de 2004.

\begin{tabular}{|c|c|c|c|c|c|c|}
\hline Variáveis & $\left.P^{1}\right)$ & $\begin{array}{l}\text { MC } \\
\text { (média } \pm D\end{array}$ & $\left.P^{1}\right)$ & $\begin{array}{l}\text { MT } \\
\text { (média } \pm \mathrm{D}\end{array}$ & $\left.P^{1}\right)$ & $\begin{array}{l}\text { Total } \\
\text { (média } \pm \mathrm{D}\end{array}$ \\
\hline $\begin{array}{l}\text { Peso ao nascimento }(\mathrm{g}) \\
\text { Comprimento }(\mathrm{cm}) \\
\text { Idade gestacional (semanas) } \\
\text { Apgar no } 1^{\circ} \text { minuto } \\
\text { Apgar no } 5^{\circ} \text { minuto }\end{array}$ & 222 & $\begin{array}{l}1556 \quad \pm \\
40 \pm 2,1 \\
32 \pm 1,7 \\
7 \pm 2 \\
9 \pm 1\end{array}$ & 245 & $\begin{array}{l}1560 \pm \\
40 \pm 2,3 \\
32 \pm 2,3 \\
6 \pm 2 \\
9 \pm 1\end{array}$ & 233 & $\begin{array}{l}1558 \quad \pm \\
40 \pm 2 \\
32 \pm 2 \\
7 \pm 2 \\
9 \pm 1\end{array}$ \\
\hline
\end{tabular}

'DP: desvio-padrão; $\mathrm{MC}=$ método canguru; $\mathrm{MT}=$ método tradicional.

Em relação às características maternas, a idade das mães de bebês do grupo MC variou de 17 a 43 anos, com valores em média \pm o desvio-padrão de $30 \pm 6$ anos, enquanto que a de mães do grupo MT, variou de 19 a 40 anos, com valores de $29 \pm 6$ anos; na amostra total 70 (58\%) eram primigestas; 75 (62\%) eram primíparas e, 8 (7\%) já haviam sofrido, pelo menos, um aborto. Quando avaliamos os grupos separadamente, encontramos 38 (63\%) de primigestas no grupo MC e 32 (53\%) no grupo MT, 40 (67\%) de primíparas no grupo MC e 35 (58\%) no grupo MT, $3(5 \%)$ de ocorrência de aborto no grupo MC e $5(8 \%)$ no grupo MT.
Dos 60 RNPT que receberam assistência pelo Método Tradicional, apenas 19 (32\%) nasceram em período anterior à implantação do Método Canguru, e os demais, 41 (68\%), não realizam o contato pele-apele, por indisponibilidade materna, visto que esta assistência, diante da estabilidade clínica do bebê, era oferecida a todas as mães, que poderiam optar livremente.

No que se refere aos diagnósticos clínicos, além da prematuridade comum a todos os bebês estudados, encontramos $28 \%$ de gemelaridade no grupo MC e $45 \%$ no MT, algum grau de desconforto respiratório em $40 \%$ dos bebês no $\mathrm{MC}$ e $51 \%$ no $\mathrm{MT}$, $22 \%$ de amniorrexe prematura no MC e $12 \%$ no MT e 
$12 \%$ dos bebês eram considerados pequenos para a idade gestacional no grupo MC e $22 \%$ no MT.

Tabela 3- Descrição do peso e duração da internação dos recém-nascidos pré-termo assistidos pelo Método Canguru e pelo Método Tradicional numa maternidade privada de Ribeirão Preto, no período de janeiro de 2002 a junho de 2004

\begin{tabular}{lcc}
\hline \multicolumn{1}{c}{ Variáveis } & $\begin{array}{c}\text { MC } \\
\left(\text { média } \pm D P^{1}\right)\end{array}$ & $\begin{array}{c}\text { MT } \\
\left(\text { média }^{1} \mathrm{DP}^{1}\right)\end{array}$ \\
\hline Peso ao nascimento & $1556 \pm 222$ & $1560 \pm 245$ \\
Peso na alta hospitalar & $2013 \pm 165$ & $1991 \pm 178$ \\
Ganho de peso diário (g/dia) & $15,8 \pm 5,4$ & $14,9 \pm 6,0$ \\
Ganho de peso (g/kg/dia) & $10,7 \pm 4,6$ & $10,2 \pm 5,0$ \\
Duração da internação (dia) & $27,3 \pm 8,4$ & $26,2 \pm 12,5$ \\
Permanência em UTI (dia) & $15,7 \pm 12,1$ & $13,1 \pm 14,1$ \\
\hline
\end{tabular}

'DP: desvio-padrão; $\mathrm{MC}=$ método canguru; $\mathrm{MT}=$ método tradicional.

$\mathrm{Na}$ Tabela 4 apresentamos os resultados obtidos com a estratificação da amostra de acordo com a idade gestacional.

Tabela 4 - Evolução do peso e duração da internação de acordo com a idade gestacional dos recém-nascidos pré-termo assistidos pelo Método Canguru e pelo Método Tradicional numa maternidade privada de Ribeirão Preto, no período de janeiro de 2002 a junho de 2004.

\begin{tabular}{|c|c|c|c|c|c|c|}
\hline \multirow[t]{2}{*}{ Variável } & \multicolumn{2}{|c|}{$\mathrm{IG} \leq 30$} & \multicolumn{2}{|c|}{$30<I G<34$} & \multicolumn{2}{|c|}{$I G \geq 34$} \\
\hline & $\mathrm{MC}$ & $\mathrm{MT}$ & $\mathrm{MC}$ & MT & $\mathrm{MC}$ & MT \\
\hline $\begin{array}{l}\text { Peso de } \\
\text { Nascimento (g) }\end{array}$ & $1382 \pm 185$ & $1338 \pm 256$ & $1629 \pm 202$ & $1650 \pm 182$ & $1541 \pm 219$ & $1662 \pm 156$ \\
\hline $\begin{array}{l}\text { Peso na alta } \\
\text { (g) }\end{array}$ & $2105 \pm 269$ & $2048 \pm 216$ & $1993 \pm 125$ & $1999 \pm 204$ & $1974 \pm 76$ & $1938 \pm 90$ \\
\hline $\begin{array}{l}\text { Ganho de peso } \\
\text { (g/dia) }\end{array}$ & $19,3 \pm 3,1$ & $16,7 \pm 5,7$ & $13,8 \pm 4,9$ & $13,6 \pm 5,9$ & $17,5 \pm 6,4$ & $14,6 \pm 6,2$ \\
\hline $\begin{array}{l}\text { Ganho de peso } \\
\text { Relativo } \\
\text { (g/kg/dia) }\end{array}$ & $14,3 \pm 2,8$ & $13,4 \pm 5,2$ & $8,9 \pm 4,0$ & $8,6 \pm 4,4$ & $12,0 \pm 5,4$ & $9,0 \pm 4,3$ \\
\hline $\begin{array}{l}\text { Tempo de } \\
\text { internação }\end{array}$ & $36,6 \pm 5,9$ & $39,6 \pm 10,2$ & $25 \pm 7$ & $23,5 \pm 9,5$ & $24,3 \pm 7,5^{\star}$ & $17,7 \pm 6,2$ \\
\hline Tempo de UTI & $28 \pm 10$ & $24,3 \pm 16$ & $13,3 \pm 10,8$ & $11,8 \pm 11,3$ & $9,8 \pm 9,2$ & $5,0 \pm 7,1$ \\
\hline
\end{tabular}

* diferença estatisticamente significante $(p<0,05)$ entre os grupos MC e MT para idade gestacional $\geq 34$ semanas;

IG: idade gestacional; MC: Método Canguru; MT: Método Tradicional.

$\mathrm{Na}$ Tabela 5 apresentamos os resultados obtidos com a estratificação da amostra de acordo com o peso de nascimento. 
Tabela 5 - Evolução do peso e duração da internação, de acordo com o peso de nascimento nos recém-nascidos pré-termo assistidos pelo Método Canguru e pelo Método Tradicional numa maternidade privada de Ribeirão Preto, no período de janeiro de 2002 a junho de 2004.

\begin{tabular}{|c|c|c|c|c|c|}
\hline & \multirow{2}{*}{ Variáveis } & \multicolumn{2}{|c|}{ Peso $<1500 \mathrm{~g}$} & \multicolumn{2}{|c|}{ Peso $\geq 1500 \mathrm{~g}$} \\
\hline & & $\mathrm{MC}$ & MT & $\mathrm{MC}$ & MT \\
\hline & $\begin{array}{l}\text { Peso de } \\
\text { nascimento }\end{array}$ & $1335 \pm 127$ & $1283 \pm 147$ & $1714 \pm 120$ & $1721 \pm 104$ \\
\hline 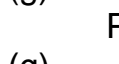 & Peso na alta & $1990 \pm 185$ & $1980 \pm 140$ & $2030 \pm 149$ & $1998 \pm 199$ \\
\hline peso & $\begin{array}{l}\text { Ganho de } \\
\text { (g/dia) }\end{array}$ & $19,4 \pm 3,5$ & $18,5 \pm 2,9$ & $13,3 \pm 5,1$ & $12,9 \pm 6,4$ \\
\hline relativo & $\begin{array}{l}\text { Ganho } \\
\text { (g/kg/dia) }\end{array}$ & $14,6 \pm 3,0$ & $14,8 \pm 3,0$ & $7,9 \pm 3,3$ & $7,6 \pm 3,9$ \\
\hline & $\begin{array}{l}\text { Tempo de } \\
\text { internação }\end{array}$ & $34 \pm 6,3$ & $37,4 \pm 10$ & $22,7 \pm 6,4^{*}$ & $19,7 \pm 8,6$ \\
\hline & Tempo UTI & $21,0 \pm 10,5$ & $20,1 \pm 15,8$ & $12,0 \pm 12$ & $9,0 \pm 11,3$ \\
\hline
\end{tabular}

\section{DISCUSSÃO}

Quando se analisou a amostra, nas características maternas, encontraram-se entre as mães dos bebês em assistência tradicional, 25 (42\%) que tinham um filho ou mais aguardando por elas em casa, contra $20(33 \%)$ entre as mães dos bebês assistidos pelo Método Canguru. Verificou-se também, que entre estas mães havia mais ocorrência de abortos anteriores, em relação às mães dos bebês assistidos pelo Método Canguru, cinco (8\%) contra três $(5 \%)$, respectivamente.

A interação entre alguns fatores, tais como perda de um bebê em gestação anterior, presença de outros filhos, participação dos pais e de outros membros da família, bem como os arranjos domésticos, podem determinar padrões mais ou menos favoráveis à opção e prática do Método Canguru. A prática do Método Canguru, embora reconhecida pela mãe como fundamental para o desenvolvimento do bebê, apresenta dilemas nem sempre fáceis de administrar, principalmente quando realizado intra-hospitalar (TOMA, 2003).

Por tratar-se de uma maternidade conceituada, cuja UTI Neonatal é referência na região, recebia muitas mulheres com gestações de risco, bem como recém-nascidos de risco procedentes de outras cidades, o que muitas vezes, levava às mães, quando tinham condições financeiras e apoio familiar, a mudarem-se temporariamente para Ribeirão Preto, porém em algumas situações, estas questões eram entraves que impossibilitavam as mães de participarem como gostariam da assistência a seus bebês.
Em relação ao ganho de peso, os resultados da investigação, bem como os dados de literatura, não demonstram claramente um aumento no ganho de peso pelo uso do Método Canguru, porém indicam que se houver algum reflexo no ganho do peso, este tende a ser favorável, já que nenhum estudo realizado constatou qualquer evidência de que o Método Canguru implicasse em menor ganho de peso para o RNPT.

Alguns estudos não evidenciaram diferenças significativas no crescimento dos bebês assistidos pelo Método Canguru em relação ao Método Tradicional, durante 6 meses de acompanhamento (SLOAN et al, 1994; CHARPAK et al, 1997).

Estudo realizado em três hospitais de países diferentes evidenciou que os bebês que receberam assistência pelo Método Canguru apresentaram maior ganho de peso em comparação aos bebês assistidos pelo Método Tradicional $(21,3 \mathrm{~g}$ e $17,7 \mathrm{~g})$, porém a diferença foi estatisticamente significante apenas para a amostra total. Isoladamente, os resultados de cada hospital, apesar de serem ligeiramente maiores para - grupo MC, não tiveram significância estatística (CATTANEO et al, 1998).

Estudo de outros autores (LINCETTO et al, 1998) evidenciou um ganho de peso diário de $20 \mathrm{~g} / \mathrm{dia}$ entre os bebês assistidos pelo Método Canguru, superior aos valores encontrados neste estudo, porém deixam claro em sua metodologia que avaliaram o ganho diário a partir do início do ganho de peso, ou seja, após o período de perda de peso fisiológica, enquanto nossa avaliação deu-se a partir do peso de nascimento.

LIMA et al (2000), em estudo realizado no Recife, Pernambuco, encontrou um ganho de peso 
diário médio de 15g/dia, semelhante aos nossos achados.

$\mathrm{Na}$ literatura, vários estudos correlacionam o Método Canguru ao tempo de internação. Muitos apontam uma redução na permanência hospitalar do RNPT assistido pelo MC, porém há que se ressaltar que vários desses estudos têm em comum a utilização do Método Canguru em período integral, inclusive após a alta, sendo comum a alta precoce, até como meio para a liberação de leito hospitalar (CATTANEO et al, 1998; CHARPAK et al, 1997; LEGAULT \& GOULET, 1995). Os achados neste estudo em relação ao tempo de internação, diferem destes resultados, até porque na instituição estudada, - Método Canguru era realizado exclusivamente durante a internação e por período de 2 horas. Outra diferença era quanto ao critério de alta, sendo necessário que o RNPT alcançasse um peso em torno de $2000 \mathrm{~g}$ para alta hospitalar, além de estar clinicamente estável, mantendo sua temperatura corporal e capaz de sugar eficientemente.

O estudo de ROBERT et al (2000) utilizou o MC por período semelhante ao utilizado nesta investigação e avaliou quase as mesmas variáveis embora tenha comparado o contato pele-a-pele com o mesmo período no colo, com um contato através das roupas. Os autores analisaram a utilização do contato pele-a-pele por um período mínimo de 2 horas, em que, entre outras variáveis, avaliaram ganho de peso e permanência hospitalar. Seus resultados se assemelham aos encontros neste estudo e mostram ganho de peso e tempo de permanência hospitalar semelhantes entre os dois grupos avaliados.

Um estudo conduzido sobre o MC relacionados ao tempo de internação chama a atenção para melhores resultados encontrados nos RNPT menores (CHARPAK et al, 1997).

Diante disto, a amostra do presente estudo foi estratificada de acordo com a idade gestacional e o peso de nascimento. Quando se analisam os dados obtidos subdividindo os RNPT em classes, de acordo com a idade gestacional, percebe-se que o tempo de internação manteve uma correlação direta com o tempo de permanência em UTI entre os grupos com $I G \geq 34$ semanas. Observa-se claramente que para os RNPT com idade gestacional $\geq 34$ semanas, o tempo de permanência em UTI entre os assistidos pelo Método Canguru foi maior que entre os RNPT assistidos pelo Método Tradicional (9,8 e 5,2 dias), e também o tempo de internação (24,3 e 17,7 dias), sendo inclusive, significante estatisticamente.

Contrariando esta correlação, observa-se que, entre os RNPT com idade gestacional $\leq 30$ semanas, que foram assistidos pelo Método Canguru, ainda que a permanência em UTI tenha sido ligeiramente maior, o tempo de internação foi menor (36,4 e 39,6 dias), embora esta diferença não tenha sido estatisticamente significativa.
Como acontece com os RNPT com idade gestacional menor que 30 semanas, quando se realiza esta análise para os RNPT com peso de nascimento $<1500 \mathrm{~g}$, também se encontra um tempo de internação menor no grupo MC, embora não seja estatisticamente significante.

Para os RNPT com peso $\geq 1500 \mathrm{~g}$, observa-se uma diferença estatisticamente significante na duração da internação entre os grupos MC e MT (22,7 e 19,7 dias, respectivamente), podendo este aspecto estar correlacionado ao tempo de permanência em UTI, que para o grupo do MC, foi maior (12 e 9 dias), da mesma forma evidenciada na estratificação de acordo com a idade igestacional $\geq$ 34 semanas (vide Tabela 4 e 5).

\section{CONCLUSÃO}

De acordo com os resultados obtidos, não foi constatado que o Método Canguru, realizado por um período de $2 \mathrm{~h} / \mathrm{dia}$, melhore de forma significativa o ganho de peso ou diminua o tempo de internação do recém-nascido pré-termo. Também, não ficou constatado que o Método Canguru comprometa o ganho de peso dos bebês, ou seja, o responsável pelo prolongamento do tempo de internação, porém acredita-se que estudos prospectivos, objetivando diminuir o número de variáveis intervenientes, devam ser desenvolvidos para elucidar seus reais benefícios no crescimento somático dos RNPT e, principalmente, os possíveis benefícios para os recém-nascidos com pesos mais baixos, avaliando, inclusive, os efeitos dessa assistência à longo prazo.

De acordo com a vivência de implantação do Método Canguru no local do estudo e com a experiência profissional na área, cabe ressaltar que o Método Canguru se mostrou viável e exeqüível em uma instituição privada, podendo ser recomendado como modelo de assistência ao recém-nascido prétermo, principalmente devido a seus inúmeros benefícios na relação entre pais e bebês já evidenciados em literatura.

O uso do Método Canguru, sem dúvida, humaniza a prática da assistência neonatal, por permitir uma maior participação da mãe e da família na assistência ao recém-nascido pré-termo de acordo com a literatura, sem comprometer o ganho de peso, conforme foi constatado pelos resultados obtidos. Portanto, cabe aos profissionais de saúde aliar todo o conhecimento à tecnologia existente e, estes, ao mais nobre de todos os sentimentos, permitindo que os recém-nascidos pré-termo desfrutem, junto a sua família, dos cuidados especializados que necessitem, de forma mais humana, buscando restituí-los o mais rápido possível ao seu núcleo familiar com qualidade de vida.

\section{REFERÊNCIAS BIBLIOGRÁFICAS}


CANO, A.; FONS, F.; BRINES, J. The effects on offspring of premature parturition. Hum. Reprod. Update, v.7, n.5, p.487-494, 2001.

CATTANEO, A. et al. Kangaroo mother care for low birthweight infants: a randomized controlled trial different settings. Acta Pediatr., v.87, n.9, p.976-985, 1998.

CHARPAK, N. et al. Kangaroo Mother versus traditional care for newborn infants< or $=2000$ grams: a randomized, controlled trial. Pediatrics., v.100, n.4, p.682-688, 1997.

CHARPAK, N.; FIGUEROA, Z. de C.; RUIZ, J.G. The Bogota declaration on kangaroo mother care: Conclusions at the Second International Workshop on the Metod. [on line]. Disponível em: http://www.blackwell-

synergy.com/doi/pdf/10.1111/i.1651-

2227.2000.tb03365.x. [Acesso em 8 jun. 2004].

CUST, A.E.; DARLOW, B.A.; DONOGHUE, D.A; Australaina and New Zealand neonatal network. Outcomes for high risk New Zealand newborn infants in 1998-1999: a population based, national study. Arch. Dis. Child Fetal Neonatal., v.88, n.1, p.15-22, 2003.

DOLE, N. et al. Maternal stress and preterm birth. Am. J. Epidemiology, v.157, n.1, p.14-24, 2003.

GENOVESE, R.E. Conhecimento, aceitação e disponibilidade dos profissionais de saúde sobre o método mãe canguru em unidade neonatal de um hospital universitário do município de São Paulo.2001. 86f. Dissertação (Mestrado). Escola Paulista de Medicina, Universidade Federal de São Paulo, São Paulo.

LEGAULT, M.; GOULET, C. Comparision of kangaroo and traditional methods of removing preterm infants from incubators. JOGNN, v.24, n.6, p.501-56, 1995.

LIMA, G.;QUINTERO-ROMERO, S.; CATTANEO, A. Feasibility, acceptability and cost of Kangaroo mother care in Recife, Brazil. Ann.Trop.Paediatr.,v.20, n.1, p.22-26, 2000.

LINCETTO,E.T. et al. Impact of season and discharge weight on complications and growth of Kangaroo mother care treated low birth weight infants in Mozambique. Acta Paediatr., v.87, p.433-439, 1998.

MINISTERIO DA SAÚDE - BNDES - FUNDAÇÃ̃O ORSA. Informativo método mãe canguru. n.6, 2002. ROBERT, K.L.; PAYNTER, C.; McEWAN, B. A comparison of Kangaroo mother care and conventional cuddling care. Neonatal Netw, v.19, n.4, p.31-35, 2000.

SLOAN, N.L.; CAMACHO, L.W.; ROJAS, E.P.; STERN, C. Kangaroo mother method: randomised controlled trial of an alternative method of care for stabilised low-birthweight infants. Maternidad Isidro Ayora Study Team. Lancet, v. 344, n.8932, p.13041305. 1994.

TOMA, T.S. Método Mãe-Canguru: o papel dos serviços de saúde e das redes familiares no sucesso do programa. Cad. Saúde Pública, v.19, p.233-242. Rio de Janeiro, 2003.
WAGNER, M.B.; PETRILLO, V.; GAY, V. A prevalence of survey of nosocomial infection in Brazilian hospital. J. Hosp. Infect., v.15, n.4, p.379381, 1990.

WORLD HEALTH ORGANIZATION (WHO). Kangaroo Mother Care a practical guide [on line]. Geneva, 2003. 54p. Disponível em: http://www.who.int/reproductivehealth/publications/kmc/text.pdf. [Acesso em 20 jul. 2004].

Artigo recebido em 18.04.2006

Aprovado para publicação em 31.08.2006 